\title{
MULTI-PARAMETRICAL HARMONIZATION MODELS IN STRATEGIC MANAGEMENT
}

\author{
Avner Ben-Yair ${ }^{1}$, Dimitri Golenko-Ginzburg ${ }^{2}$, Zohar Laslo ${ }^{3}$ \\ ${ }^{1}$ Department of Industrial Engineering and Management, Sami Shamoon Academic College of Engineering, \\ Beer-Sheva, 84100, Israel, Tel.: (972)-8-6475649,E-mail:avnerb@sce.ac.il \\ ${ }^{2}$ Department of Industrial Engineering and Management (Professor Emeritus), Ben-Gurion University of the Negev, \\ Beer-Sheva, 84105, Israel, Tel.: (972)-8-6472225, E-mail: dimitri@bgumail.bgu.ac.il, and Department of Industrial \\ Engineering and Management, Academic College of Judea and Samaria, Ariel, 44837 \\ ${ }^{3}$ Department of Industrial Engineering and Management, Sami Shamoon Academic College of Engineering, \\ Beer-Sheva, 84100, Israel, Tel.: (972)-8-6475640,E-mail: zohar@sce.ac.il
}

Received 4 April 2007; accepted 15 June 2007

\begin{abstract}
The aim of this paper is to consider a company engaged in designing and creating a new product and, later on, delivering the latter in large quantities to the market. The product is composed of several sub-products, each of them, in turn, being a subject of several possible versions. The product's utility comprises both the utility of designing and creating the product's pattern example as well as the competitive utility to gain the future commercial success.

The problem is to determine the income versions of designing sub-products in order to maximize the product's competitive utility subject to restrictions related to the design process.

A two-level search algorithm for the problem's solution is suggested. The internal level is faced with optimizing the product's competitive utility by means of experts' information, while the external level centers on obtaining a routine feasible solution from the point of designing process.
\end{abstract}

Keywords: Multi-attribute utility; Competitive utility; Basic project's attributes and competitive attributes; Harmonization model on the basis of expert team's decision-making.

\section{Introduction}

It can be well-recognized (see, e.g. $[1-3,4,5])$ that existing quality techniques in various organization systems (OS) are restricted to market competitive problems only. Those techniques usually center on analysing the competitive quality of organization systems' outcome products in order to gain future commercial success. But in that capacity they ignore the quality of the OS functioning, e.g. the quality of designing and creating a new unique product. In our recent publications $[6,7]$ we developed several new utility models to estimate the project's quality in the course of its realization. However, if the company is engaged in designing and creating a new product and, later on, delivering the latter in large quantities to the market, the product's life cycle continues far beyond terminating the design process. Thus, developing quality models of organization systems in their entirety centers on developing new utility models comprising design and production phases as well as the system of divestment phase. Nowadays engineering projects where a new system or product are being designed, developed, manufactured and continuously quality tested, may span years, as applicable for the case of a new automobile, or over a decade for a nuclear power plant [5]. New product development takes anywhere from several months to several years. In lengthy processes of this type, decisions made at the outset may have substantial, long-term effects that are usually difficult to forecast. The trade-off between current objectives and long-term consequences of each decision is a strategic aspect [5] of project management. Thus, the research to be considered refers to strategic management and deals with the most important aspects of that area. Moreover, special attention is drawn in literature to life cycle costing (see, e.g. [8, 9]) in case a decision having long-term effects deals with selection of components and parts for a new system or a new product at the advanced development and detailed design phase.

Thus, an evident conclusion can be drawn that the newly developed utility harmonization models must deal not only with the quality of OS functioning, but with the quality of outcome products as well, especially when subject to severe market competition. In the paper 
under consideration an attempt will be made to enhance utility models [1-3] in order to cover the whole life cycle of the regarded product. We suggest to develop a more generalized utility harmonization model by implementing in the latter the most essential aspects of the MAUT theory [1-3, 4, 5]. The generalized harmonization model should, thus, be applied to all stages of the product life cycle, namely:

1. MAUT multi-attribute models have to undergo certain modifications to be used at the stage of designing the outcome product, on the basis of experts' decision-making in combination with proper ranking and scaling.

2. Analytical and simulation models which have been already incorporated in partial harmonization algorithms (PHM) in Safety Engineering and Project Management [6,7], should be applied at the stages of designing and creating the new device.

3. We suggest to link the outlined above MAUT and PHM together in order to optimize the process of designing and creating a new product within its entire life cycle. The suggested optimization algorithm should be of mixed type, i.e., to comprise both analytical calculations and man-computer dialogs at the stage of decision-making on the basis of experts' interviews. The backbone of the optimization algorithm is that all its elements (including analytical calculations and dialogs with experts) are parts of a generalized search procedure to determine the optimal version of the product to be designed and created.

It is assumed that the product to be manufactured is composed of several sub-products, e.g. a new automobile comprises an engine, a bonnet, a brake system, etc. Each sub-product, in turn, is a subject of several possible versions. The problem is to determine for each sub-product their optimal versions in order to maximize the product's competitive utility subject to restrictions related to the design process. A two-level optimization algorithm based on the cyclic coordinate search method (CCSM) $[6,10]$, is suggested. The internal level is faced with optimizing the product's competitive utility by means of experts' information, while the external level centers on obtaining a routine feasible solution from the point of designing process.

\section{The problem description and definitions}

In order to formalize the harmonization problem we will require some new definitions.

\section{Definitions}

Call a quantitative parameter entering the project of designing and creating a new product, the basic project attribute (BPA) together with its corresponding restriction value. The latter serves as the worst permissible value that may be implemented into the design project. Several basic project attributes may be independent as well as dependent parameters. BPA restrictive values are already pre-given by the OS management. However, in the course of carrying out the design project, they may be subject to alterations, e.g. owing to changes of the product's demand on the market.

Call a quantitative parameter entering the outcome product, i.e., the designed product to be delivered to market, the basic competitive attribute (BCA). BCA values actually form the product's competitive utility in order to gain future commercial success. Those values are usually calculated by means of expert information.

As outlined above, the system under consideration comprises:

- the phase of the product's designing and creating the pattern example, and

- later on, the second phase related to delivering the product in large quantities to the market.

It can be well-recognized that both BPA and BCA values depend on the set of versions assigned to each subproduct. Note that BPA values are fully determined by the set of versions, i.e., those values can be calculated analytically or by means of simulation. BCA values are calculated through expert information taking into account the set of versions as well. Assume, further, that when benefiting from commercial success, the profit obtained from delivering the product to the market at the second phase, usually exceeds essentially the project's expenses to design the product at the first phase. Thus, we suggest to develop a multi-attribute utility value on the basis of only BCA values. This generalized value has to be maximized in the course of the suggested CCSM algorithm by means of information obtained from experts. As to BPA values, they have to be incorporated in the search procedure in order to satisfy the pre-given restrictions.

Referring to MAUT models [1-3, 4, 5], we will assume that for each BCA value two opposite estimates have to be pre-given before carrying out the design process:

- the least preferred value having practically very poor chances to win the market competition, and 
- the most preferred value which enables the attribute to win the competition.

Note that both opposite estimates for each competitive attribute can be obtained from the expert team on the basis of interview questions. Those estimates play the leading part in the process of questioning experts to obtain the multi-attribute utility values $[1-3,4,5]$.

In the model under consideration we will develop a modification of the classical MAUT procedure of both questioning experts and obtaining utility values. This is because the existing MAUT expert models cannot be incorporated in a search harmonization procedure.

\section{The suggested expert interview procedure to calculate multi-attribute values}

As outlined above, the suggested general idea to maximize the multi-attribute utility value centers on undertaking a search procedure in the multi-dimensional state of possible combinations - possible versions assigned to sub-products. The suggested search procedure is carried out by implementing the cyclic coordinate descent method where each $i$-th coordinate varies from 1 to $r_{i}$, $r_{i}$ being the number of possible versions which can be assigned to the $i$-th sub-product.

Let the number of sub-products be $q$. Thus a routine search point is actually a $q$-dimensional vector $\vec{D}=\left(d_{1}\right.$, $d_{2}, \ldots, d_{q}$ ) with integer numbers. In the course of undertaking the search procedure vector $\vec{D}$ has to satisfy all pre-given BPA restrictions (let them be $m$ ), otherwise the routine search point is not considered. If all BPA restrictions are honored, search point $\vec{D}$ has to be passed and later on examined by a team of experts, by means of the following interview procedure. Let the expert team comprise $f$ experts faced with the problem of decision-making on $n$ competitive attributes. It is assumed that (before examining any routine point $\vec{D}$ ) for each basic competitive attribute BCA two opposite estimates: the least competitive and the most competitive ones $-\mathrm{BCA}^{*}$ and $\mathrm{BCA}^{* *}$ - are already determined.

Each expert $E g, 1 \leq g \leq f$, after receiving the interview questions, examines and analyzes carefully the input information $\vec{D}$ and for each $k$-th recurrent competitive attribute $B C A_{k}$ gives his personal subjective judgment on:

1. The expert's expected value of $B C A_{k}$, which will be henceforth designated as $B C A_{g k}$; note that
- estimating $B C A_{g k}$ is carried out always for a concrete set of versions assigned to the sub- products, i.e., for the routine search point $\vec{D}$;

- value $B C A_{g k}$ has to be always placed between the corresponding pre-given opposite estimates $B C A_{k}{ }^{*}$ and $B C A_{k}{ }^{* *}$.

2. The expert's estimated value (order) of importance of attribute $B C A_{k}$ to win the competition for the product on the market. Denote henceforth this order of importance by $\eta_{g k}$.

After obtaining the answers from all experts we suggest to modify values $B C A_{g k}, 1 \leq g \leq f, 1 \leq k \leq n$, to their relative equivalents $\gamma_{g k}$ as follows:

$\gamma_{g k}=\frac{B C A_{g k}-B C A_{k}^{*}}{B C A_{k}^{* *}-B C A_{k}^{*}}$

Note that relation (1) does not undergo any changes, both in case relation $B C A_{k}{ }^{* *}>B C A_{k}{ }^{*}$ holds, or otherwise. Value $\gamma_{g k}$ represents, in essence, the relative competitive ability of the routine set of versions $\vec{D}$ due to attribute $B C A_{k}$ only. Thus, relation

$\sum_{k=1}^{n}\left(\gamma_{g k} \cdot \eta_{g k}\right)=W_{g}$

denotes the subjective judgment of the $g$-th expert about the total value of the product's competitiveness. We suggest to calculate the more generalized estimate, which we will henceforth call the product's competitive utility

$U_{C}=\frac{1}{f} \sum_{g=1}^{f} W_{g}=\frac{1}{f} \sum_{g=1}^{f} \sum_{k=1}^{n}\left(\gamma_{g k} \cdot \eta_{g k}\right)$.

Value $U_{C}$ calculated by (3) is just the parameter which has to be maximized in the course of implementing the search algorithm. Note that while using the CCSM algorithm, the number of feasible search points to be examined is less than by implementing other methods. Thus, the number of interview questions to the expert team will be diminished as much as possible.

Note, in conclusion, that according to the MAUT models, pairwise comparisons have to be undertaken by experts, in cases, when dependencies between two or more competitive attributes take place [1-3]. Those techniques may also be used by experts in our models, in the course of determining competitive attributes $B C A_{k}$. However, other techniques involving subjective judgments, can be implemented as well [4]. 


\section{Notation}

Let us introduce the following terms:

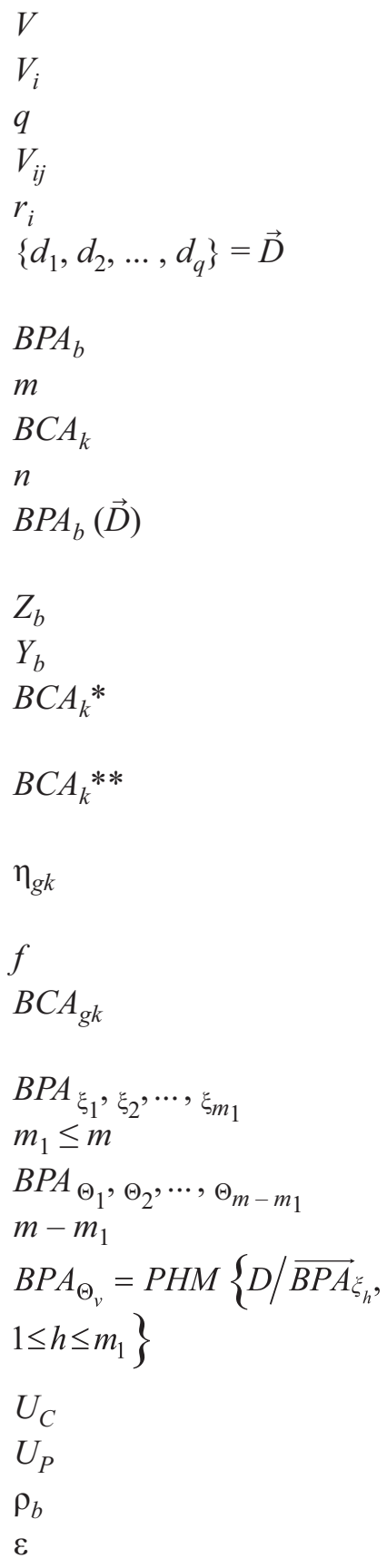

- the product to be designed and manufactured;

- the $i$-th sub-product entering the product, $1 \leq i \leq q$;

- the number of sub-products;

- the $j$-th possible version of the $i$-th sub-product, $1 \leq j \leq r_{i}$;

- the number of possible versions of sub-product $V_{i}$;

- a routine search point (a routine set of versions) for the CCSM algorithm, $1 \leq d_{i} \leq r_{i}, 1 \leq i \leq q$;

- the $b$-th basic project attribute value, $1 \leq b \leq m$;

- number of BPA values;

- the $k$-th basic competitive attribute value, $1 \leq k \leq n$;

- number of BCA values;

- the $b$-th basic project attribute value calculated analytically or by means of simulation at the routine search point $\vec{D}$;

- the worst permissible value for the $b$-th basic project attribute (pre-given);

- the best possible value for the $b$-th basic project attribute, $1 \leq b \leq m$ (pre-given);

- the worst competitive estimate of the $k$-th competitive attribute (pre-given by experts);

- the best competitive estimate of the $k$-th competitive attribute (pre-given by experts);

- priority level (level of importance) of the $k$-th competitive attribute given by the $g$-th expert, $1 \leq g \leq f$;

- the number of experts entering the team;

- the personal subjective judgment of the $g$-th expert on the expected value of the $k$-th competitive attribute;

- basic independent project attributes (pre-given);

- number of independent basic project attributes (pre-given);

- basic dependent project attributes (pre-given);

- number of dependent project attributes;

- the estimate of basic project dependent parameters, $1 \leq v \leq m-m_{1}$, obtained by means of implementing partial harmonization models or simulation models on the basis of vector $\vec{D}$;

- the product's multi-attribute competitive utility (to be maximized);

- the project's utility obtained by means of BPA values;

- partial utility value for the $b$-th BPA (pre-given);

- the relative accuracy of the harmonization problem's objective (value $U_{C}$ ).

\section{The problem formulation}

Referring to Sections 2-3, the strategic harmonization problem is as follows: to determine optimal versions assigned to all sub-products $d_{1}^{(o p t)}, d_{2}^{(o p t)}, \ldots, d_{q}^{(o p t)}$, to maximize the multi-attribute competitive utility value

$\operatorname{Max} U_{C}$

$\{\vec{D}\}$

s.t. $\min \left(Y_{b}, Z_{b}\right) \leq B P A_{b}(\vec{D}) \leq \max \left(Y_{b}, Z_{b}\right), 1 \leq b \leq m$,

where $U_{C}$ satisfies (3).

Restriction (5) means that only feasible solutions $\vec{D}$, i.e., sets of versions which honor pre-given worst permissible constraints $Z_{b}$, can participate in the optimization procedure.

We suggest to solve the strategic harmonization problem (3-5) by means of a two-stage algorithm. At the 
first stage feasible solutions $\vec{D}$, i.e., combinations of versions assigned to the sub-products, are determined. Those vectors present input information for the second stage, to maximize the multi-attribute competitive utility parameter $U_{C}$ obtained by means of experts subjective judgments.

Note that in some cases it might be not easy to develop the initial feasible search point $\vec{D}$ at the first stage. We suggest to implement in the algorithm the corresponding subsidiary Problem $A I$ which can be formulated as follows:

Determine at least one combination $\vec{D}=\left\{d_{1}, d_{2}, \ldots, d_{q}\right\}$ satisfying restriction (5).

\section{Subsidiary problem AI}

The suggested step-wise algorithm to solve Problem $A I$ is as follows:

Step 1. By means of the Monte-Carlo method simulate for each sub-product the index of its version, i.e., simulate integer values

$d_{i}=\left[\alpha_{i} \cdot r_{i}\right]+1,1 \leq i \leq q$,

where $\alpha_{i}=U(0,1)$ is a random value uniformly distributed in $(0,1)$, and $[x]$ is the whole number of $x$.

Step 2. By means of Monte-Carlo simulate for each $i$-th sub-product the values of $m_{1}$ independent basic project attributes

$$
\begin{aligned}
& B P A_{\xi_{h}}=\min \left(Y_{\xi_{h}}, Z_{\xi_{h}}\right)+\alpha_{h} . \\
& \left\{\max \left(Y_{\xi_{h}}, Z_{\xi_{h}}\right)-\min \left(Y_{\xi_{h}}, Z_{\xi_{h}}\right)\right\}, \\
& 1 \leq h \leq m_{1}, \\
& \text { where } \alpha_{h}=U(0,1) .
\end{aligned}
$$

Step 3. Using partial harmonization models [10-11], determine values of $m-m_{1}$ dependent basic project attributes $B P A_{\Theta_{v}}=P H M\left\{\vec{D} / B P A_{\xi_{h}}\right.$, $\left.1 \leq h \leq m_{1}\right\}$. In case $m=3$ there are usually two independent basic attributes (time to accomplish the project and budget assigned to the project) as well as one dependent attribute - reliability for the project to be accomplished on time. In such a case the problem together with the corresponding algorithm is outlined in [1-2]. If $m$ exceeds 3 , the problem becomes more complicated.

Step 4. If all values $B P A_{\Theta_{v}}=P H M\left\{\vec{D} / B P A_{\xi_{h}}\right\}, 1 \leq$ $v \leq m-m_{1}$, satisfy (5), search point ${ }^{2} \vec{D}$ obtained at Step 1 is a feasible one, thus providing solution to the problem. Otherwise apply the next step.
Step 5. Repeat Steps 2-3 $N_{1}$ times, in order to check possible combinations in different $m$ - dimensional subspaces. If for a significantly large $N_{1}$ a feasible solution has still not been obtained, apply the next step.

Step 6. Repeat Steps 1-5 $N_{2}$ times, where $N_{2}$ is a significantly large number. If no feasible solution has been obtained, the initial search point cannot be determined. We have either to alter values $\left(Y_{b}, Z_{b}\right), 1 \leq b \leq m$, or to select other possible versions of the sub-products.

We have deliberately chosen the so-called undirected Monte-Carlo search method [11] because of its simplicity. If $m$ is not large, using the method for solving Problem AI does not cause any particular difficulties.

\section{Cyclic coordinate search method for the problem solution}

To obtain the problem solution, we suggest to implement the CCSM in the two-level optimization algorithm. The step-wise algorithm is as follows:

Step 1. Solve subsidiary Problem AI to obtain a feasible problem solution, i.e., determine vector $\vec{D}$ which will be used henceforth as the initial search point.

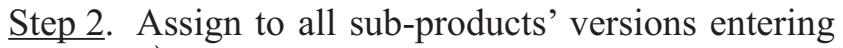
$\vec{D}$ (obtained at Step 1), the minimal index 1, i.e., $\vec{D}=(1,1, \ldots, 1)$. For each sub-product $i$, $1 \leq i \leq q$, all other versions can be enumerated in an arbitrary order from 2 to $r_{i}$.

Step 3. Transfer the information about the initial search point, i.e., the set of sub-products' versions, to the expert team. After carrying out questioning interviews and receiving the experts' subjective judgments, calculate value $U_{C}$ by (3). Coordinates of vector $\vec{D}$ together with value $U_{C}$ are placed in a special array $W$. In the course of the optimization process, this array will contain the monotonously increasing utility value $U_{C}$ together with the corresponding vector of optimized variables $\vec{D}$. Denote henceforth the stored information by $U_{C}{ }^{*}$ and $\vec{D}^{*}$, correspondingly.

Step 4. Start using CCSM with respect to the coordinate variables $d_{1}, d_{2}, \ldots, d_{q}$, beginning from the initial search point $\vec{D}=(1,1, \ldots, 1)$. 
The general idea is to increase the first coordinate $d_{1}$ by a constant step equal 1 , i.e., $d_{1, j}=$ $d_{1, j-1}+1$, while all other coordinates $d_{2}, \ldots, d_{q}$ are fixed and remain unchanged in the course of the coordinate optimization. After the first coordinate $d_{1}$ is optimized, we fix the index of the latter, and proceed by increasing the second coordinate $d_{2}$ by a step equal 1 (coordinates $d_{3}, \ldots, d_{q}$ being fixed). Afterwards, when completing optimization of the second coordinate, the latter is fixed as well, and we proceed with the third coordinate $d_{3}$, and so forth, until all coordinates are looked through by the partial coordinate increasing procedure. Go to Step 5.

Step 5. Proceed with the optimization process starting again from the first coordinate $d_{1}$ with the index obtained in the course of carrying out Step 4. Check two opposite directions: $d_{1}-1 \Rightarrow d_{1}$ and $d_{1}+1 \Rightarrow d_{1}$ and choose one of them which results in obtaining a feasible solution as well as in increasing utility value $U_{C}$. If such a direction can be chosen, proceed changing $d_{1}$ in that direction by a constant step equal 1 . The same procedure has to be undertaken with other coordinates. Note that, similar to Step 4, only one coordinate undergoes optimization, while all other coordinates remain fixed and unchanged.

If in the course of carrying out Steps 4-5 a routine feasible search point $\vec{D}$ coincides with the previously obtained and stored in array $W$ feasible point $\vec{D}^{*}$, the corresponding utility value $U_{C}{ }^{*}$ is taken as the quasi-optimal solution of the harmonization problem. Thus, the search process terminates. Go to Step 9 .

Implementing the optimization search process at Steps 4-5 centers on numerous applications to a group of Steps 6-7, which actually examine the routine search point and carry out decision-making as follows:

- either to accept the routine search point as a successful one, i.e., to proceed with the search procedure from that point on, or

- to reject the routine search point and change the optimizing coordinate.

As outlined above, the coordinate optimization centers on examining a routine search point $\vec{D}$ in order to check:

- the search spoint's feasibility, and

- the increase of the corresponding total utility $U_{C}$ relatively to the previously obtained maximal value $U_{C}{ }^{*}$.
In order to check the feasibility, apply the next step.

Step 6. To check the routine search point's feasibility, one has to carry out Steps 2-5 of Problem AI outlined in Section 6. Note that coordinate values $d_{1}, d_{2}, \ldots, d_{q}$ enter the routine search point $\vec{D}$.

If in the course of carrying out Step 6 a feasible solution has been obtained, go to the next step. Otherwise reject the routine search point and go to Step 8.

Step 7. Undertake questioning interviews of experts and, after obtaining their subjective judgments, calculate value $U_{C}$ by (3). If $U_{C}$ exceeds $U_{C}{ }^{*}$ (stored in array $W$ ), examine the relative increase of the utility value by calculating

$\Delta U_{C}=\frac{1}{U_{C}^{*}}\left(U_{C}-U_{C}^{*}\right)$

If relation $\Delta U_{C}<\varepsilon$ holds, the optimization process terminates. Go to Step 9. Otherwise, when $\Delta U_{C} \geq \varepsilon$ holds, accept the routine search point as a successful one. Go to Step 4, to continue the search procedure.

If $U_{C}$ does not exceed $U_{C}{ }^{*}$, the routine search point has to be rejected. Go to the next step.

Step 8. Assume that in the course of the optimization search process, the $i$-th coordinate, i.e., value $d_{i}$, has increased its index by 1 , while other values $d_{1}, \ldots, d_{i-1}, d_{i+1}, \ldots, d_{q}$ were fixed and remained unchanged. If $i \neq q$, decrease value $d_{i}$ by $1, d_{1}-1 \Rightarrow d_{1}$, fix value $d_{i}$ and start optimizing the next coordinate $d_{i+1}$. Go to Step 4 .

If $i=q$, i.e., all coordinates have been partially optimized, the process is then repeated starting with $d_{i}$ again. Go to Step 5 .

Step 9. The optimization search process terminates, and the information stored in array $W$, i.e., $\vec{D}^{*}$ with objective $U_{C}{ }^{*}$, is taken as the optimal solution of the harmonization problem.

\section{Case of compound utility}

It can be well-recognized that in previous sections the competitive utility value $U_{C}$ has been favored over the project utility value $U_{P}$. However, under certain conditions those different utility parameters may be regarded as practically of equal importance. Thus, the problem of maximizing the competitive utility value $U_{C}$ has to be substituted for maximizing the compound utility 
$\beta_{P} \cdot U_{P}+\beta_{C} \cdot U_{C}=U_{T}$,

where $U_{T}$ is the total utility value and $\beta_{P}$ and $\beta_{C}$ are properly chosen coefficients to present both utility parameters in similar ranking and scaling.

The problem is, thus, to determine the optimal set of versions for each sub-product $\vec{D}$ in order to maximize the total utility $U_{T}$

$$
\underset{\{\vec{D}\}}{\operatorname{Max}} U_{T}=\underset{\{\vec{D}\}}{\operatorname{aax}}\left\{\beta_{P} \cdot U_{P}+\beta_{C} \cdot U_{C}\right\}
$$

subject to (5), where $U_{C}$ is calculated by (3) and $U_{P}$ satisfies $[6,7]$

$U_{P}=\sum_{b=1}^{m}\left(\rho_{b} \cdot B P A_{b}\right)$

We suggest to optimize harmonization model $(3,5$, 9-11) by using the same search algorithm as being outlined in Section 7. Only minor modifications have to be implemented, namely:

1. Step 1 has to be substituted by the algorithm of solving Problem AII to maximize the project's utility value

$$
\underset{\{\vec{D}\}}{\operatorname{Max}} U_{P}=\underset{\{\vec{D}\}}{\operatorname{Max}}\left[\sum_{b=1}^{m}\left(\rho_{b} \cdot B P A_{b}\right)\right]
$$

subject to (5).

Problem $(5,12)$ together with the corresponding algorithm, has been outlined in our recent publications $[6,7]$, especially for the three-attribute harmonization model in project management.

2. Step 6 has to be substituted by solving problem (5, 11) as well.

3. Competitive utility value $U_{C}{ }^{*}$ in array $W$ has to be substituted by the total utility value $U_{T}^{*}$.

4. Relation (8) has to be substituted by another one, honoring modification (9).

All other steps of the algorithm do not undergo any changes.

\section{Example on designing a new passenger vehicle}

An example on designing a new passenger vehicle which is widely presented in the literature on project management (see, e.g. [5]), can illustrate implementation of the outlined above harmonization model. The example is subject to restrictions by competitive attributes which have been used within a long period by questioning experts and obtaining from the latter all kinds of subjective decision-making. However, no harmonization models have been suggested and no optimization problems have been solved.

Three basic attributes define usually the $R \& D$ project's utility:

$B P A_{1}$ - budget assigned to the whole project;

$B P A_{2}$ - time to accomplish the project;

$B P A_{3}$ - reliability for the project to be accomplished on time on condition of pre-given $B P A_{1}$ and $\mathrm{BPA}_{2}$.

Thus, there are two independent attributes $\left(B P A_{1}\right.$ and $\left.B P A_{2}\right)$ and a dependent one $\left(B P A_{3}\right)$. For the case of a PERT-COST project the harmonization model together with the optimization algorithm obtained a detailed solution in [7].

As to competitive attributes, nine of them have been singled out [5] and are usually examined by experts in case of designing a new vehicle:

$B C A_{1}$ - relative fuel economy;

$B C A_{2}$ - initial cost;

$B C A_{3}$ - life cycle cost per mile;

$B C A_{4}$ - maintainability (special scaling);

$B C A_{5}$ - safety (special scaling);

$B C A_{6}$ - refuel time;

$B C A_{7}$ - unrefueled range;

$B C A_{8}$ - maximum startup time;

$B C A_{9}$ - minimum speed-up time from 0 to $80 \mathrm{mph}$.

As to the worst and the best competitive attribute estimates, they are as follows (for the last decade - based on best experts' opinion):
1. $B C A_{1}^{*}=20 \mathrm{mpg}$ equivalent; $B C A_{1}^{* *}=80 \mathrm{mpg}$ equivalent;
2. $B C A_{2}{ }^{*}=\$ 25,000$; $B C A_{2}^{* *}=\$ 5,000$;
3. $B C A_{3}{ }^{*}=\$ 1,00 / \mathrm{mile}$; $B C A_{3} * *=\$ 0,20 / \mathrm{mile}$
4. $B C A_{4}^{*}=0$ (special scaling); $B C A_{4}{ }^{* *}=10$ (special scaling);
5. $B C A_{5}{ }^{*}=0$ (special scaling); $B C A_{5}{ }^{* *}=10$ (special scaling);
6. $B C A_{6} *=8$ hours; $B C A_{6}{ }^{* *}=0,17$ hours;
7. $B C A_{7}^{*}=50$ miles; $B C A_{7}{ }^{* *}=250$ miles;
8. $B C A_{8}{ }^{*}=600$ seconds; $B C A_{8}^{* *}=5$ seconds;
9. $B C A_{9}{ }^{*}=60$ seconds; $B C A_{9}{ }^{* *}=5$ seconds. 
It can be well-recognized that an experienced decisionmaker is capable of undertaking subjective judgment for any $B C A_{k}, 1 \leq k \leq 9$, together with scaling the attribute's level of importance $\eta_{k}$.

\section{Conclusions and future research}

The following conclusions can be drawn from the study:

1. The problem of maximizing the product's utility by means of considering optimal components for that product, is widely regarded in the literature as an important strategic area in project management. Thus, developing new harmonization models on that subject refers to strategic harmonization models. Those models practically cover the entire life cycle of any newly designed and developed product.

2. The backbone of the models under consideration is the generalized search procedure comprising partially harmonization models on the basis of heuristic approaches with decision-making on the competitive ability of the designed product, to be undertaken by a qualified expert team.

3. The suggested search algorithm is based on the cyclic coordinate search method. The latter may either comprise:

- an optimization procedure in the area of basic competitive attributes only, in order to maximize the total competitive utility subject to restrictions for basic parameters of the designing project, or

- a search procedure to maximize the total product's utility comprising as summants both the competitive utility and the project's utility values.

The two outlined above procedures are not of any principal difference; one can be obtained from another by implementing only minor modifications.

4. As far as we are concerned similar research has not been undertaken as yet.

5. An emphasis has to be drawn that, in dependence on the novelty of the designed product, the market's demands, etc., other variables to be optimized may be introduced in the harmonization model. However, the basic concepts linked to the necessity of developing a mixed type optimization procedure comprising a combination of heuristic methods and interview dialogs with experts, have to remain unchanged.

6. Future research to develop new strategic harmonization models has to be undertaken not only in project management, but in additional areas of strategic management as well.

\section{Acknowledgment}

This research has been partially supported by the Paul Ivanier Center on Robotics and Production Research, Ben-Gurion University of the Negev.

\section{References}

1. KEENEY, R. L. The art of assessing multi-attribute utility functions. Organizational Behavior and Human Performance, 1997, 19, p. 267-310.

2. KEENEY, R. and RAIFFA, H. Decisions with multiple objectives: preferences and value tradeoffs. Wiley, New York, 1976.

3. KEENEY, R. and WINTERFELDT, D. van. Eliciting probabilities from experts in complex technical problems. IEEE Transactions on Engineering Management, 1991, 38 (3), p. 191-201.

4. OSTER, S. Modern competitive analysis. $2^{\text {nd }}$ edition, Oxford University Press, New York, 1999.

5. SHTUB, A.; BARD, J. and GLOBERSON, Sh. Project management: engineering, technology and implementation. Prentice Hall International, Inc., New York, 1994.

6. BEN-YAIR, A. Harmonization models in strategic management and safety engineering. $\mathrm{Ph}$. D. Thesis, BenGurion University of the Negev, Beer-Sheva, Israel, 2004.

7. BEN-YAIR, A. and MENIPAZ, E. Three-parametrical harmonization model in project management by means of simulation. Mathematics and Computers in Simulation, 2002, 59 (5), p. 431-436..

8. DHILLON, B. S. Life cycle costing: techniques, models and applications. Gordon and Breach, New York, 1989.

9. FABRYCKY, J. W. and BLANCHARD, B. S. Life cycle cost and economic analysis. Prentice Hall, Englewood Cliffs, New Jersey, 1991.

10. LUENBERGER, D. G. Introduction to linear and nonlinear programming. Addison Wesley Publishing Co., Massachusetts, 1973.

11. MENIPAZ, E. Essentials of production and operations management. Prentice Hall, Englewood Cliffs, New Jersey, 1973. TAHA, H. A. Operations research: an introduction. MacMillan, New York, 1987. 